\section{Eine Gesundheitskarte für die Schweiz}

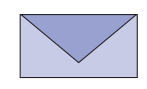

Ein Herr M. Beng, Dipl. Ing., ETH, Siemens Schweiz AG, versucht, uns Ärzte für dumm zu verkaufen [1]. Mit Eloquenz preist er diverse Versuche mit elektronischen Gesundheitskarten als alleinseligmachende, notwendige Errungenschaften an.

Meine Fragen an ihn:

- Wer übernimmt die horrenden Kosten für die Einführung und Weiterbearbeitung dieser Kärtchen und der Bearbeitungsstationen?

- Wer kann länderübergreifend einheitlichen Standard garantieren? (Sofern mit unseren Schriftzeichen verfasst, kann ich bei jedem aus $\mathrm{x}$-welchem Land Hierhergereisten die wichtigsten Angaben entziffern, keine elektronische Karte kann so was je bieten.)

- Hat sich der Herr überlegt, warum «auf Mann» getragene Karten bei vielen Zahlungsversuchen kläglich versagen?

- Hat er überlegt, was in 10 oder gar 20 Jahren mit all den gespeicherten Daten passiert, wenn kein Gerät mehr die dann antiquierten Zeichen lesen kann (alle Elektronik ist nach 5 Jahren veraltet und nach 10 Jahren Schrott). Meine 35jährigen, papiernen Krankengeschichten geben noch jetzt Auskunft über Befunde, während weder 30jährige Mikrofilme noch elektronische 15jährige Dateien mehr einlesbar sind.

- Wer ist verantwortlich, wenn irgendein wichtiger Befund oder eine Verordnung nicht in die Karte eingetragen ist? Selbst Banales wie z.B. die Blutgruppe ist mit heutiger Analysetechnik gar nicht mehr banal, Nebenwirkungen z. B. aufgrund von CYP sind erst in den letzten Jahren klar geworden.

Sicherheitsdispositiv und sektorieller Datenschutz sind bei einer für jedermann zugänglichen Karte sowieso illusorisch.

\section{Fazit}

Die vorgeschlagene Gesundheitskarte ist in der Praxis noch unbrauchbarer als der ICD-Code und gefährdet sogar die Gesundheit des Trägers. Statt eines Spareffektes führte sie bei flächendeckender Anwendung zu erheblichen Mehrkosten.

Dr. med. J. Riediker, Effretikon für die Schweiz. Schweiz Ärztezeitung 2005;86(37):2139-4.

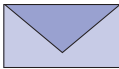

Herr Beng publizierte einen sehr informativen Artikel über die mögliche Einführung der Gesundheitskarte in der Schweiz. Aus Sicht der praktizierenden Ärztinnen und Ärzte scheint uns jedoch ein Kommentar wichtig:

- Der Artikel betont vor allem das grosse Potential einer Gesundheitskarte im Rahmen einer serverbasierten Lösung mit elektronischer Patientenakte. Allerdings wird auch auf die hohen Einführungskosten (Milliarden) für eine solche Lösung hingewiesen. Als Beispiel wird die Einführung eines Kartensystems in der italienischen Region Lombardei mit etwa 9 Mio. Einwohnern angeführt. Für Gesundheitskarte und Kartenmanagementsystem zeichnete die Firma Siemens verantwortlich, bei welcher auch der Autor arbeitet. Der Beitrag stammt somit von einem Experten der Industrie. $\mathrm{Zu}$ einigen zentralen Aussagen fehlen leider Referenzen.

- Eine Suche im elektronischen Archiv der SÄZ unter den Stichworten «Gesundheitskarte, Patientenkarte, Versichertenkarte» fördert 19 Beiträge zutage. Zumeist sind es Artikel, in welchen das Stichwort lediglich im Rahmen anderer Ausführungen zu Themen um E-Healthcare erwähnt wird. Manche davon sind von Martin Denz, dem ehemaligen E-Health-Beauftragten der FMH, verfasst worden. Andere stammen aus der Feder von Technikern, Public-Health-Fachleuten oder Ökonomen. Kein einziger Beitrag - von zwei Leserbriefen abgesehen - ist von praktizierenden Kollegen verfasst oder mitverfasst worden. Seit dem Abgang von Martin Denz aus der FMH herrscht Funkstille von ärztlicher Seite. Angesichts der bevorstehenden Umwälzungen ist dies erschreckend.

- Aus der Praxis und der Literatur ist bekannt, dass die Informatisierung der Arztpraxen nur dann einen Nutzen entfalten kann, wenn die Arbeitsprozesse konsequent darauf ausgerichtet und angepasst werden [2]. Mit anderen Worten: Der Einzug der Informatik in die Praxen und Sprechzimmer geht mit grossen Änderungen einher. Wer die nötigen Anpassungen herunterspielt, streut uns Ärztinnen und Ärzten Sand in die Augen. Dieser Einzug in unsere Praxen findet statt, ob wir das wollen oder nicht. Auf das Wie könnten und sollten wir jedoch entschieden Einfluss nehmen.

- Uns sticht unter anderem der Satz «der Kontakt eines Patienten mit einem Arzt, sowohl in einer ärztlichen Praxis wie in einer Klinik, wird sich damit nur wenig verändern» ins 
Auge. In der hausärztlichen Praxis versuchen wir, dem Modell der «patientenzentrierten Konsultation» nachzuleben, weil dies dem Charakter der Arzt-Patienten-Beziehung am ehesten entspricht und in der Regel auch die besten, weil die Kooperation fördernden Ergebnisse erbringt. Die vom Autor vorgestellte elektronische Patientenakte mit hinterlegten Datenbanken und Dialogführung ist demgegenüber eine dritte Instanz im Sprechzimmer. Sicher kann sie zum Nutzen der Patienten eingesetzt werden, was aber einen langwierigen Lern- und Bewusstseinsprozess voraussetzt. Die IT-Experten vertreten meist ein völlig anderes Denkmodell, das unseren Anforderungen nicht gerade entgegenkommt. Auch in Ländern mit einer fortgeschritteneren Informatisierung der Praxen steht die Optimierung der Instrumente für die Sprechstunde erst am Anfang [3].

Was ist die Quintessenz? Die Entwicklung der Informationstechnologie hat unser Arbeitsumfeld in den Kliniken, aber auch in den Praxen schon fang, die kommenden Veränderungen werden weit einschneidender sein. Die Gesundheitskarte ist in diesem Prozess ein Schlüsselinstrument. Viele Fragen z.B. um Datenschutz und den Aufwand der Datenpflege stehen erst am Anfang einer Diskussion. Weder die Patientenseite noch die betroffenen Berufsgruppen sind bisher in diesem Prozess angemessen vertreten.

- Fazit 1: Informationen ja gerne, wenn möglich aber ohne Beschönigungen.

- Fazit 2: Wollen wir als Ärzteschaft hier mitreden oder uns weiterhin der Initiative von Ämtern, Konzernen und Kostenträgern überlassen?

Heinz Bhend, SGAM.Informatics

Marco Zoller, Verein compIS

2 Miller RH. California Health Care Foundation. Electronic Medical Records: Lessons from Small Physician Practices. San Francisco: University of California; 2003.

3 de Lusignan S. What is primary care informatics? J Am Med Inform Assoc 2003;10:304-9. Sehr geehrte Kollegen

Besten Dank für Ihre Äusserungen im Zusammenhang mit dem Artikel zur Gesundheitskarte. Sie stellen in Ihren Zuschriften wichtige und dringende Fragen zur Diskussion: Wie werden diese Karten - und vor allem und in viel grösserem Ausmass - die dahinterliegenden Datenbearbeitungskonzepte die ärztliche Arbeit mit den Patienten beeinflussen? Wo findet sich Potential zur Verbesserung der Betreuungsprozesse? Was kostet die Einführung einer solchen Karte? Und last but not massiv beeinflusst. Dies war jedoch erst der An-

Der Zentralvorstand hat sich in den vergangenen Monaten verschiedentlich mit diesen Fragen befasst, dies allerdings im Sinne einer fundierten Meinungsbildung bisher eher im Hintergrund. Angesichts des zunehmenden Aktivismus der Bundesbehörden wie auch einer nachgerade naiven Technikbegeisterung vor allem auch der Industrie (neudeutsch: der «Solution Provider») hat der FMH-Zentralvorstand entschieden, dass der Zeitpunkt für eine breite Diskussion dieser Themen innerhalb der Ärzteschaft jetzt gekommen ist.

Inhaltlich teilt der Zentralvorstand nicht alle Positionen der Absender, anerkennt aber den Vorwurf einer bislang unbefriedigenden, da unklaren Trennung zwischen der Versichertenkarte gemäss KVG Art. 42a, welche ausschliesslich administrative Daten enthält, und weiterführenden Konzepten, bei welchen medizinische Daten des Patienten auf der Patientenkarte gespeichert werden sollen - von den medizinischen Leistungserbringern notabene. Dass dies hohe Zusatzinvestitionen für die Aufrüstung der Arztpraxen bedeutet, ist klar - und hier schweigt des (Bundes-)Sängers Höflichkeit ...

Wer nun der Meinung anhängen sollte, dass eine reine Erhöhung der Anzahl Personalcomputer, Tablet-PCs, ADSL-Anschlüsse, Router, Switches usw. die Einführung einer Patientenkarte in der Arztpraxis sicherstellen kann, irrt sich. Die Umstellung auf eine elektronische Patientendokumentation («elektronische KG») - und darauf läuft eine effizient umgesetzte und korrekt funktionierende Patientenkarte mit medizinisch relevanten Inhalten schlussendlich hinaus - ist eine gewaltige Herausforderung nicht nur an uns Ärztinnen und Ärzte, sondern auch an die Patientinnen und Patienten, die Behörden und last but not least - die Politik. Da geht's nicht mehr um einen Wechsel des Mediums für die Dokumentation der stattgefundenen Patientenkontakte, sondern um eine fundamentale Umstellung von Kernprozessen in der Beziehung zwischen Patient und Arzt bzw. Ärztin. Eine Patientenkarte stellt zudem ein Steuerungsinstrument für das Patientenverhalten dar.

Lassen Sie mich klarstellen: in der erwähnten least: cui bono - wer profitiert davon?
Umstellung liegt durchaus ein beträchtliches Potential, unser Gesundheitsversorgungssystem weiterzuentwickeln - auch und gerade im Interesse der Patienten und Patientinnen. Dies ist allerdings nicht gratis zu haben und kann nur dann erfolgreich sein, wenn sich alle Beteiligten aktiv in die Diskussion einbringen können und so sichergestellt wird, dass die weitere Entwicklung «en connaissance des causes» erfolgt.

Ludwig T. Heuss, Mitglied des Zentralvorstandes der FMH 